\title{
I linguaggi della storia: Storia \& Musica
}

L'articolo propone un resoconto della rassegna organizzata dall'Istituto storico della Resistenza e dell'età contemporanea di Parma sul rapporto tra la storia e la musica. In un dialogo tra musicisti e storici si è cercato di mettere a fuoco le problematiche che l'uso della fonte musicale pone allo storico, considerando anche il possibile uso didattico delle opere prese in esame.

This article is a report of a series of conferences organized by Istituto storico della Resistenza e dell'età contemporanea di Parma in order to analyze a medium such as popular music, the way it mingles with historical issues and the question whether it may represent a reliable source for historians. In a dialogue between musicans and historians, the meetings' aim has been to focus on the questions about the meaning that this language can represent for historians and the teaching of history.

I cori delle mondine nelle risaie in Veneto; il tempo dei lavori forzati cadenzati dai blues negli Stati Uniti; i canti partigiani; l'Italia del boom che si ferma ipnotizzata davanti all'esibizione di Domenico Modugno a Sanremo; le canzoni della contestazione globale degli anni Sessanta.

La musica ha accompagnato la storia dell'uomo fin dai suoi albori, dai riti misterici fino ad arrivare al consumo di musica leggera a partire dalla metà del Novecento per arrivare allo streaming che oggi rende possibile ascoltare tanto Monteverdi quanto la musica nepalese: questa forma di arte e di comunicazione però non si è limitata ad essere colonna sonora ma è stata un vero e proprio fattore ed agente di cambiamento (o, per converso, di conservazione) della Storia.

All'interno della seconda edizione di "Linguaggi della storia", rassegna incentrata sullo studio della storia culturale con un focus particolarmente attento alle 
culture giovanili e alle fonti della cultura popolare, l'Istituto della Resistenza e dell'età Contemporanea di Parma ha deciso di dedicare l'edizione del 2019 proprio al rapporto tra storia e musica, presentando saggi di storici che indagano il filo sottile che lega le note e i testi degli artisti - non tralasciando però aspetti come il marketing e le apparizioni video dei casi presi in esame - alle società del Novecento. Gli eventi, rivolti agli insegnanti (in quanto riconosciuti come corso d'aggiornamento) ma anche alla cittadinanza generica, sono stati appositamente dislocati in sedi "non conferenziali" ma in luoghi della città che proprio dall'integrazione tra musica e socialità traggono tuttora la loro linfa vitale: il pub Tonic, il circolo Arci Zerbini, il Circolo Giovane Italia e la Casa della musica.

L'incontro d'apertura ha visto come protagonista un genere musicale, il jazz, in un contesto del tutto particolare come quello dell'Italia fascista. A raccontarlo è stata Camilla Poesio, autrice del volume Tutto è ritmo, tutto è swing. Il jazz, il fascismo e la società italiana [Poesio 2018], in dialogo con Marco Minardi (Isrec Parma). A partire dal suo testo, Poesio ha toccato una serie di temi a lungo rimasti ignorati dalla storiografia intorno al fascismo che invece il tema del jazz consente di sviscerare, permettendo anche di riconsiderare certi "luoghi comuni" sulla dittatura mussoliniana: come il suo carattere totalmente autarchico e il rifiuto totale di una modernità proveniente da oltreoceano. Lo studio di un genere musicale, e quindi della società che ne fruisce, rivela come il fascismo stesso fosse diviso tra «spinte conservatrici, sostenitrici di un nazionalismo musicale, poi chiamato autarchia musicale, e una visione cosmopolita» [Poesio 2018, 2]: si ha così l'immagine di una dittatura che sembra rigettare tutto ciò che la modernità americana detta "barbarica" sembra proporre [Poesio 2018, 16], ma che poi sfrutta proprio il jazz nella sua versione "italica" come strumento di consenso. Lo studio di questo particolare genere musicale permette quindi di approfondire $\mathrm{i}$

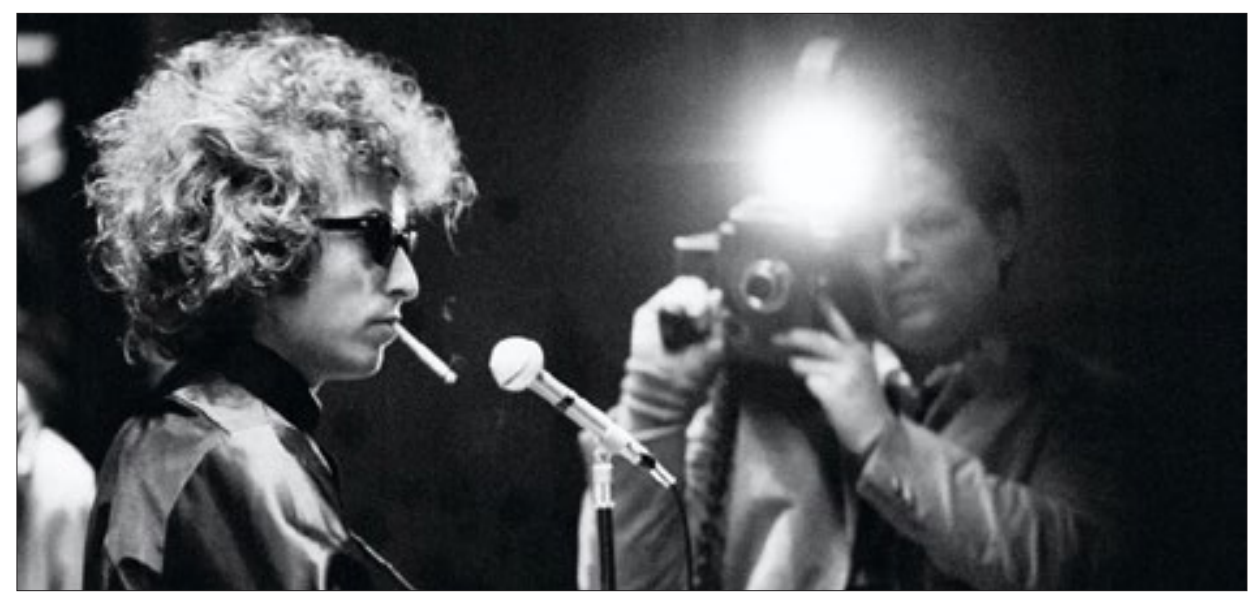

Fig.1 Bob Dylan 
modelli culturali dei consumatori di jazz e di come il regime totalitario si ponesse nel campo dell'intrattenimento. La musica proveniente da New Orleans innesca inoltre nell'Italia fascista i germi di una rivoluzione generazionale dei consumi e dei rapporti di genere (le flappers), che sembra anticipare il fermento che poi sfocerà nella contestazione degli anni Sessanta.

E proprio a una canzone di questo decennio è dedicato il secondo incontro: introdotto da Teresa Malice (Isrec Parma), Alessandro Portelli ha portato a Parma uno spettacolo-narrazione, accompagnato da Piero Brega, Susanna Buffa e Sara Modigliano, ispirato al suo Bob Dylan, pioggia e veleno. "Hard Rain", una ballata tra tradizione e modernità [Portelli 2018].

Ed è proprio nell'oscillare tra questi due poli, tra presente e passato che Portelli ha raccontato il capolavoro dylaniano e le sue matrici culturali: dal Testamento dell'avvelenato, canzone dell'Italia settentrionale del 1629 passando per la ballata anglo-scozzese Lord Randall, canonizzata nel The English and Scottish Popular Ballads verso la fine dell'Ottocento. Nella trasmigrazione culturale di modelli culturali viene così accentuata e rilevata la diversa percezione del mondo di riferimento delle società che tali modelli recepiscono: in una versione della canzone suonata nel Sud Italia il testamento del titolo lascia in eredità solo gli occhi per piangere - simbolo di una società rurale arretrata -, in quella delle regioni settentrionali nel contesto di una famiglia urbana e aristocratica viene invece lasciata una proprietà. Dalle ballate scozzesi, Portelli rintraccia come la canzone sia trasmigrata nel contesto americano e fatta propria da uno dei più grandi e controversi song-writer statunitensi: Bob Dylan. Hard Rain diventa così una canzone

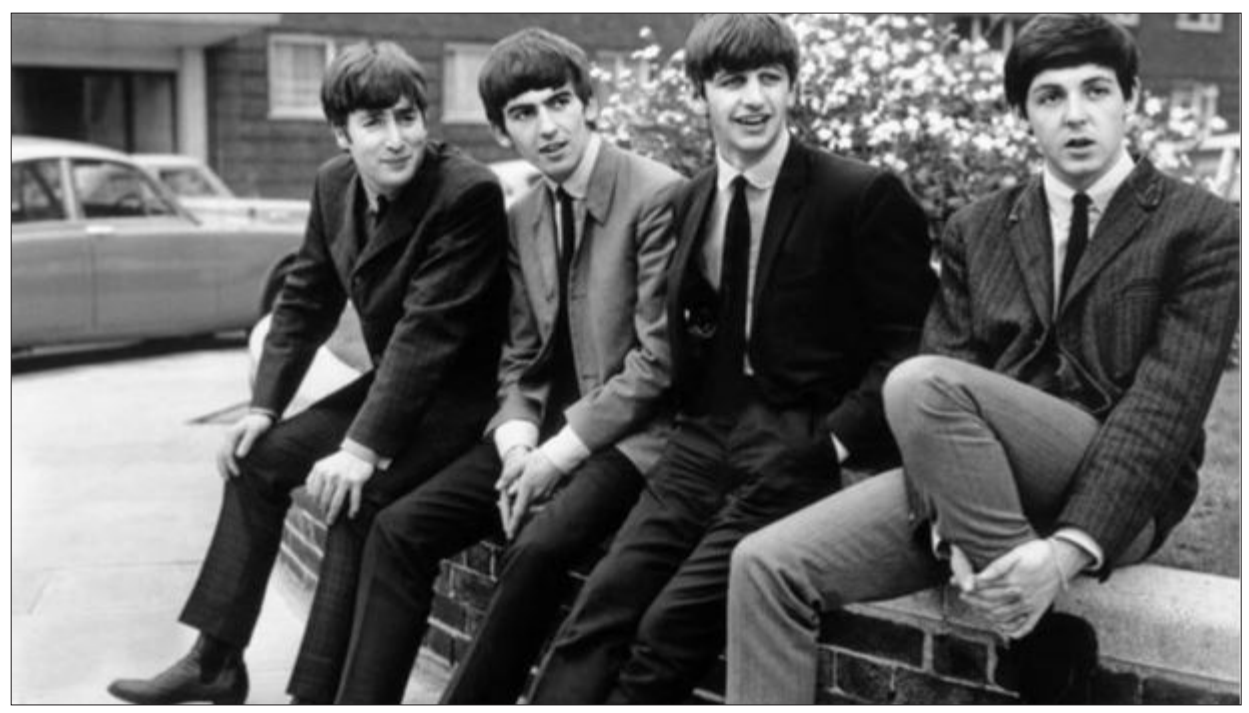

Fig. 2 Beatles 
manifesto degli anni Sessanta, ambientata nell'universo poetico del cantautore dominato da sentimento di apocalisse nucleare tipico del mondo schiacciato dalla Guerra fredda e che tuttora conserva la sua forza evocativa tanto da diventare l'inno della conferenza di Copenhagen sul clima del 2009, nonostante fu Bob Dylan stesso a negare il rapporto tra la sua canzone e la minaccia atomica.

Il terzo incontro è stato incentrato sulla band forse più importante di sempre, sia per qualità musicale sia per impatto sociale: i Beatles, raccontati dall'americanista Ferdinando Fasce, autore del volume La musica nel tempo. Una storia dei Beatles [Fasce 2018], in dialogo con Carlo Ugolotti (Isrec Parma). Le vicende dei Fab Four permettono di ricostruire innanzitutto un fenomeno sociale e culturale di inedita potenza sulle masse del Novecento ma anche, grazie alla narrazione di Fasce basata su documenti di archivio, una storia dei quattro di Liverpool come "produttori" e "consumatori" di musica.

Dalle origini in una Liverpool, sopravvissuta alla guerra ma crocevia e porto di mille culture, per arrivare allo scoppio della beatlesmania e alla Swinging London: vediamo mutare un mondo culturale, un mondo politico e un mondo di consumi in cui è proprio la cultura popolare a rendere le masse soggetti storici individuali [Chambers e Gilroy 1995]. Lo stile di vita bealtlesiano, amplificato anche da moda, cinema e riviste di settore, diventa prima di tutto una dichiarazione di "appartenenza simbolica" di una generazione che rifiuta il conformismo tradizionalista e si crea un proprio spazio di produzione e consumo culturale.

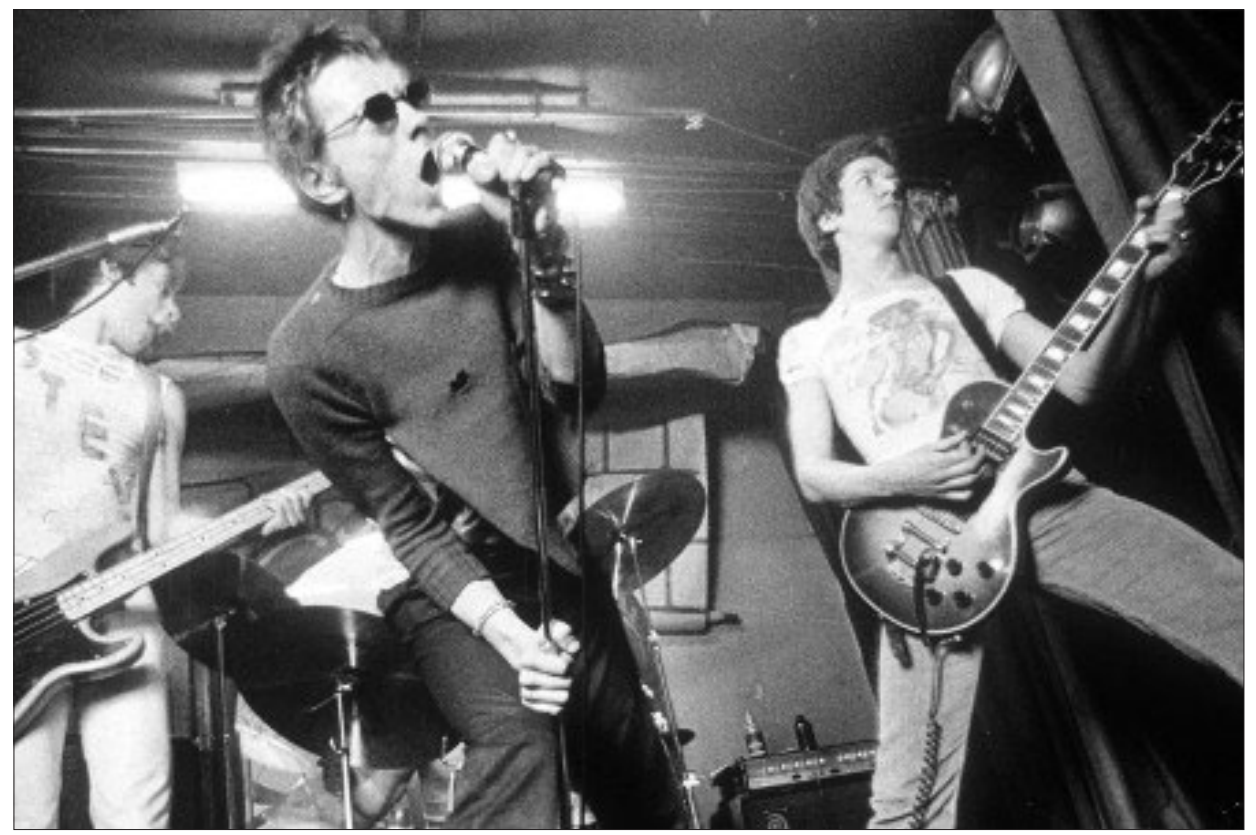

Fig. 3 Sex Pistols 
Dagli esordi fino allo scioglimento, il racconto di Fasce ha permesso di fare luce su protagonisti di primo piano della storia novecentesca, non solo musicale ma anche sociale e politica nel senso più stretto del termine (si veda il ruolo della tournée dei Beatles nel Sud segregazionista degli Stati Uniti).

Dalle utopie psichedeliche di Yellow Submarine la rassegna Isrec si è spostata per l'intervento finale ai più cupi e problematici anni Settanta del punk grazie alla narrazione di Alessia Masini, autrice di "Siamo nati da soli". Punk, rock e politica in Italia e Gran Bretagna (1977-1984) [Masini 2019], in dialogo con Domenico Vitale (Isrec). L'analisi di Masini vuole combattere la damnatio memoriae che la narrazione collettiva e una storiografia "distratta" hanno fatto calare sugli anni Ottanta: semplificati in eredi della catastrofe sociale e politica degli anni Settanta, sfociata in un generale riflusso, epitome di un rifiuto generalizzato per la politica e della scomparsa di una dimensione di partecipazione collettiva soppiantata dal consumo di un «nuovo miracolo economico».

Il discorso sul riflusso ha dunque solo considerato una pars destruens delle nuove culture giovanili, sganciandole dalla forza mitopoietica che il Punk ha saputo ispirare nelle generazioni del post ' 77 . Citando Masini: «le controculture non rappresentavano più un'alternativa alla politica, ma uno strumento della politica» [Masini 2018, 201].

Dal Jazz (o "giazzo", come lo ribattezò il fascismo), passando per Dylan e Lennon, e arrivando al Punk dei gruppi italiani: la rassegna Isrec ha provato a mostrare come tra "i linguaggi della storia" la musica sia stata uno dei fattori determinanti per la nascita delle identità sociali del Novecento.

Chiaramente in una serie di incontri così non potevano risultare assenti le note stesse e pertanto si ringraziano i musicisti che con il loro rigore (più o meno filologico) hanno dato ritmo alle narrazioni: Beppe di Benedetto e Leo Caligiuri (per il Jazz); Piero Brega, Susanna Buffa e Sara Modigliani (per Dylan); Alberto Padovani ed Enrico Fava (per i Beatles); Stefano Pavone, Francesco Scaglioni e Francesco Mazzera (per il Punk).

\section{Bibliografia}

Arcagni S., Cucco P. e Michelone G. 1998. Il cinema dei Beatles, Alessandria: Falsopiano Chambers I. e Gilroy P. 1995. Hendrix, hip-hop e l'interruzione del pensiero, Genova: Casta \& Nolan (ed. or. 1995)

De Matteis S., Lombardi M. e Somaré M. (eds.) 1980. Follie del varietà. Vicende memorie, personaggi 1890-1970, Milano: Feltrinelli 
Favaro R. e Pestalozza L. (eds.) 1996. La musica nella Germania di Hitler, 1933-1945: l'emigrazione interna, Lucca: Libreria musicale italiana

Fasce F. 2018. La musica nel tempo. Una storia dei Beatles, Torino: Einaudi

Hobsbawm E.J. 1982. Storia sociale del jazz, Roma: Editori Riuniti (ed. or. 1952)

Masini A. 2018. L'Italia del riflusso e del punk (1977-1984), Meridiana, 92

Masini A. 2019. "Siamo nati soli”. Punk, rock e politica in Italia e Gran Bretagna (19771984), Ospedaletto: Pacini Editore

Poesio C. 2018. Tutto è ritmo, tutto è swing. Il jazz, il fascismo e la società italiana, Firenze: Le Monnier Università - Mondadori Education

Portelli A. 2018.Bob Dylan, pioggia e veleno. "Hard Rain”, una ballata fra tradizione e modernità, Roma: Saggine 